\title{
Lattice model for kinetics and grain-size distribution in crystallization
}

\author{
Mario Castro* \\ GISC, Departamento de Física de Materiales, Universidad Complutense, E-28040 Madrid, Spain \\ Angel Sánchez \\ GISC, Departamento de Matemáticas, Universidad Carlos III de Madrid, E-28911 Leganés, Madrid, Spain
}

Francisco Domínguez-Adame

GISC, Departamento de Física de Materiales, Universidad Complutense, E-28040 Madrid, Spain

(Received 9 September 1999)

\begin{abstract}
We propose a simple, versatile, and fast computational model to understand the deviations from the wellknown Kolmogorov-Johnson-Mehl-Avrami kinetic theory found in metal recrystallization and amorphous semiconductor crystallization. Our model describes in detail the kinetics of the transformation and the grain size distribution of the product material, and is in good agreement with the available experimental data. Other morphological and kinetic features amenable of experimental observation are outlined, suggesting directions for further validation of the model.
\end{abstract}

\section{INTRODUCTION}

Mechanical, electronic, or magnetic properties of many polycrystalline materials depend not only on their chemical composition, but also on the kinetic path of these materials toward the nonequilibrium state. Recently, the interest on thin-film transistors made of polycrystalline $\mathrm{Si}$ and $\mathrm{Si}-\mathrm{Ge}$ grown by low-pressure chemical vapor deposition has been driven by the technological development of active matrix addressed flat-panel displays ${ }^{1}$ and thin-film solar cells. ${ }^{2}$ With these and similar applications in mind, the capability to engineer the size and geometry of grains becomes crucial to design materials with the required properties.

In general, crystallization of most materials takes place by a nucleation and growth mechanism: ${ }^{3}$ Nucleation starts with the appearance of small atom clusters (embryos). At a certain fixed temperature, embryos with sizes greater than a critical one become growing nuclei; otherwise, they shrink and eventually vanish. Such a critical radius arises from the competition between the surface tension, $\gamma$, and the difference in free energy between the amorphous and crystalline phases, $\Delta g$, that favors the increasing of grain volume, yielding an energy barrier that has to be overcome to build up a critical nucleus. For a circular grain of radius $r$, the free energy takes the simple form

$$
\Delta G=2 \pi r \gamma-\pi r^{2} \Delta g .
$$

The free energy $\Delta G$ has a maximum, the energy barrier, at the critical radius $r^{*}=\gamma / \Delta g$. Subsequently, surviving nuclei $\left(r>r^{*}\right)$ grow by incorporation of neighboring atoms, yielding a moving boundary with temperature-dependent velocity that gradually covers the untransformed phase. Growing grains impinge upon each other, forming a grain boundary, and growth ceases perpendicularly to that boundary. Therefore, the structure consists of vertices connected by edges (grain boundaries), which surround the grains. The number of edges joined to a given vertex is 3 . In some cases, at high temperatures these boundaries move until they reach a more favorable equilibrium configuration (in two dimensions, the equilibrium angles at a vertex are $\left.120^{\circ}\right) .{ }^{4}$

In the past few years, the belief that this picture is far too simple to properly describe nucleation-driven crystallization has progressively spread among the researchers in the field. This is chiefly due to two problems: On the one hand, this theory of nucleation and growth predicts an energy barrier much larger than the experimental one, implying that nucleation would be hardly probable at available annealing temperatures. ${ }^{5}$ On the other hand, it is known that in crystallization of $\mathrm{Si}$ over $\mathrm{SiO}_{2}$ substrates, nucleation develops in the $\mathrm{Si} / \mathrm{SiO}_{2}$ interface due to inhomogeneities or impurities that catalyze the transformation. ${ }^{6}$ Therefore, a theory of homogeneous nucleation and growth is not entirely applicable to the referred experiments as well as to other examples reported in the literature. ${ }^{7}$

In addition to the difficulties above, it is clear that the transformation kinetics is also problematic. It is generally accepted that the fraction of transformed material during crystallization, $X(t)$, obeys the Kolmogorov-Johnson-MehlAvrami (KJMA) model, ${ }^{8}$ according to which

$$
X(t)=1-\exp \left(-a t^{m}\right)
$$

where $a$ is a nucleation- and growth-rate dependent constant and $m$ is an exponent characteristic of the experimental conditions. Two well-defined limits have been extensively discussed in the literature: When all the nuclei are present and begin to grow at the beginning of the transformation, the KJMA exponent, $m$, is equal to 2 (in quasi-two-dimensional growth like thin films), and the nucleation condition is termed site saturation. The product microstructure is tessellated by the so-called Voronoi polygons (or Wigner-Seitz cells). On the contrary, when new nuclei appear at every step of the transformation, $m=3$ and the process is named continuous or homogeneous nucleation. Plots of $\log [-\log (1$ $-X)$ ] against $\log (t)$ (called KJMA plots) should then be 
straight lines of slope $m$. Although in some cases, the KJMA theory explains correctly the transformation kinetics, its general validity has been questioned in the last few years, ${ }^{9,10}$ and several papers have been devoted to understand this question in different ways. ${ }^{11-13}$ However, there are still some open questions: An exponent between 2 and 3 is experimentally obtained (between 3 and 4 in three dimensions) ${ }^{7}$ the KJMA plots from experimental data do not fit to a straight line in some cases ${ }^{7}$ and, finally, the connection between geometrical properties (grain size distributions) and the KJMA exponent is not clear.

In this paper we report on a detailed investigation of a probabilistic lattice model, which relates in a clear-cut way the mentioned problems to the inhomogeneities in the sample, i.e., the fact that heterogeneous nucleation takes place. Indeed, heterogeneous nucleation is rather common in nature due to impurities or substrate cavities resulting from roughness, among others. Within our model, the connection between such heterogeneous nucleation and the deviations from the simplest nucleation picture become evident. Furthermore, as we will see below, our model predicts measurable quantities, such as the grain size distribution or the KJMA exponent, which are in good agreement with the experiments. Our paper is organized according to the following scheme: in Sec. II we introduce our model and discuss in depth the relationship between its defining parameters and physical ones. Section III collects the results of an extensive simulation program, which establishes the main features of the model. Finally, Sec. IV discusses the connection between our model and experiments, and concludes the paper by summarizing our main findings and collecting some prospects and open questions.

\section{THE MODEL}

\section{A. Evolution rules}

Our model is based in some previous ideas by Cahn ${ }^{14,15}$ and Beck, ${ }^{16}$ and its key proposal is that the material is not perfectly homogeneous but, on the contrary, it contains regions with some extra energy (regions with some order produced during deposition or substrate impurities) at which nucleation is more probable. Our aim in this section is to provide a detailed description of our model (largely expanding the preliminary, short report presented earlier in Ref. 17), and how the basic idea mentioned above is implemented in it.

The model is defined on a two-dimensional lattice (square and triangular lattices were employed with essentially similar results) with periodic boundary conditions; generalizations can straightforwardly be done to any spatial dimension. In the beginning $(t=0)$, every lattice site (or node) $\mathbf{x}$ belongs to a certain grain or state. We represent the situation at $\mathbf{x}$ by $q(\mathbf{x}, t)=0,1,2, \ldots$, the state 0 being that of an untransformed region. The lattice spacing is therefore the experimental resolution, usually greater than $r^{*}$. Following the idea that the amorphous phase has random regions at which nucleation is favored, we choose a fraction $c$ of the total lattice sites and label those as able to nucleate. We term these energetically favorable sites potential nuclei.

Simulation proceeds in discrete time steps of duration $\tau$. The system evolves by parallel updating according to the following three rules and considering that initially all the material is untransformed, i.e., $q(\mathbf{x}, 0)=0$ for all lattice sites:

An already transformed site remains at the same state forever.

An untransformed potential site may become a new nonexisting state (i.e., crystallizes) with probability $n$ (nucleation probability) if and only if there are no transformed nearest neighbors around it.

An untransformed site (including potential sites) transforms into an already existing transformed state with probability $g$ (growth probability) if and only if there is at least one transformed site of that type on its neighborhood. The new state is randomly chosen among the neighboring grain states, if there are more than one.

Note that we have termed $g$ as growth probability and not growth rate. The actual growth rate is a nontrivial function $f(g)$, because when $g<1$ the grains grow with a rough boundary. For the model parameters, we expect a functional form $n \sim e^{-E_{n} / k_{B} T}$ and $f(g) \sim e^{-E_{g} / k_{B} T}$, where $E_{n}$ and $E_{g}$ are the energy barriers for nucleation and for growth, respectively (see below). Hence, temperature is implicit in the model parameters. We discuss these relationships in depth in the next subsections.

\section{B. Physically relevant magnitudes}

As we mentioned above, the crystalline fraction is approximately given by Eq. (2), with some exponent $m$ depending on the dimensionality and type of nucleation. Experimentally, the crystalline fraction is measured from the intensity of the peaks of x-ray diffraction of the microstructure as material transforms from the amorphous to the polycrystalline phase. In the following, we will assume that there is not any preferential direction, ${ }^{18}$ that is, $n$ is the same for all potential sites, and $g$ is the same for all grains.

The other experimentally measurable magnitude is the grain size distribution, $P(A)$, defined as the fraction of grains with a given area $A$. To compare with simulation results, we will usually plot the normalized distribution of reduced area $A^{\prime}=A / \bar{A}$, where $\bar{A}$ is the mean area:

$$
\bar{A}=\int_{0}^{\infty} A P(A) d A .
$$

This distribution changes dramatically with nucleation conditions. ${ }^{19}$ Some of the available experimental data are given in terms of the distribution of grain diameters, $P(d)$. As we will demonstrate below, this distribution is equivalent to the distribution of effective diameter $(A / \pi)^{1 / 2}$ (or simply $A^{1 / 2}$ ), which is computationally less expensive to calculate. Hence, we will present our results in terms of the effective diameter.

\section{Time and length scales}

To begin with, let us show that the potential sites, distributed randomly throughout the system, define a characteristic length given by the probability distribution of nearest neighbors. Suppose we have $N$ randomly potential sites in a $L$ $\times L$ system. The mean concentration of potential sites is $c$ $=N / L^{2}$. We may ask about the probability of finding a num- 


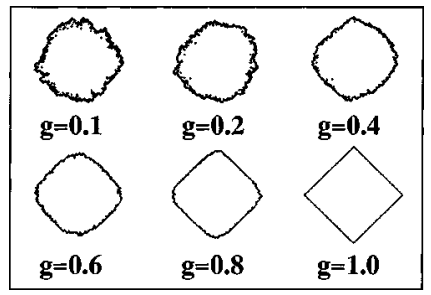

FIG. 1. Individual grains grown on a square lattice for different growth probabilities $g$.

ber $k \leqslant N$ of these sites in a region of area $A$. This probability is given by the binomial distribution:

$$
P_{k}(N, A)=\left(\begin{array}{l}
N \\
k
\end{array}\right) p^{k}(1-p)^{N-k},
$$

where $p=A / L^{2}$.

Taking the limit $L \rightarrow \infty, N \rightarrow \infty$, while keeping $N / L^{2} \equiv c$, the expression (4) tends to the Poisson probability distribution,

$$
P_{k}(A)=\frac{(A c)^{k}}{k !} e^{-A c} \text {. }
$$

If, as stated above, we suppose that the system is isotropic, we may write the probability of finding $k$ potential sites in a circle of radius $r$ as

$$
P_{k}(r)=\frac{\left(\pi r^{2} c\right)^{k}}{k !} e^{-\pi r^{2} c} .
$$

So, if Eq. (6) is the probability of finding $n$ potential sites in a disc of radius $r$, then the probability of finding no grains is

$$
P_{0}(r)=e^{-\pi r^{2} c},
$$

and the probability of finding at least one neighbor at a distance less than $r$ is $1-P_{0}(r)$. This is precisely the probability distribution of nearest neighbors. In other words, we can obtain the probability density of finding at least one neighbor between $r$ and $r+d r$ as follows:

$$
p(r) d r=\frac{d}{d r}\left(1-P_{0}(r)\right) d r=2 \pi r c e^{-\pi c r^{2}} d r .
$$

The first moment of the distribution is the mean distance among potential sites

$$
d_{m}=\int_{0}^{\infty} r p(r) d r=c^{-1 / 2}
$$

On the other hand, the grains grow with constant velocity. For definiteness, let us take the growth probability $g$ to be 1 ; we will see below that the results of simulations for other values of $g$ can be reproduced from simulations with $g=1$ conveniently rescaled. With this choice, the grain radius grows according to the law $r(t)=\Omega t$, where $\Omega$ is a geometrical coefficient that depends on the underlying lattice. Thus, we may define the mean time at which the growing grains will impinge, or overlap time, as $\Omega t_{o}=c^{-1 / 2}$ or, in general, i.e., ignoring the details of the lattice, $t_{o} \sim c^{-1 / 2}$.

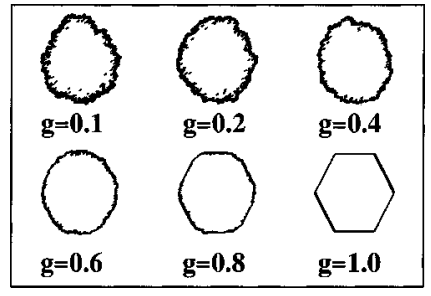

FIG. 2. Individual grains grown on a triangular lattice for different growth probabilities $g$.

The characteristic time scale arising from the concentration of nucleation sites is not the only one: Indeed, the nucleation probability defines another characteristic time. Being more specific, the number of sites that have nucleated per unit time is proportional to the available ones

$$
\frac{d N(t)}{d t}=n\left[N_{\max }-N(t)\right]
$$

where $N_{\max }=c L^{2}$. Thus, we have

$$
N(t)=N_{\max }\left(1-e^{-n t}\right) \Rightarrow \rho(t)=c\left(1-e^{-n t}\right),
$$

$\rho(t)$ being the concentration of already nucleated potential sites at time $t$. In view of this, we define the characteristic nucleation time $t_{n}=1 / n$. As we will see below, the competition between time scales characterizes the final microstructure.

In a general case, some potential sites will be covered by other growing grains and therefore their nucleation is inhibited. The mean distance of the potential sites that become actual grains is, replacing $c$ by $\rho(t)$ in Eq. (8),

$$
d_{m}(t)=\frac{1}{\sqrt{\rho(t)}}=\frac{1}{c^{1 / 2}\left(1-e^{-n t}\right)} .
$$

If $t_{n} \ll t_{o}$, almost every potential site nucleates before grains impinge upon each other. We term this situation fast nucleation, and in terms of our model parameters it means that $n \gg c^{1 / 2}$. This situation is similar to site saturation nucleation, in which every potential site nucleates at $t=0$. The KJMA exponent will be close to 2 and the grain size distribution will be similar to that of site saturation. Note that, when $n=1$, the exact limit is obtained for every concentration $c<1$, but concentrations $c$ close to 1 yield a mean grain size of just a few times the critical radius, $r^{*}$, which in fact has not much to do with the experimentally measured values. In this case, $t_{n}$ is approximately equal to the simulation time step, $\tau$, so the characteristic time scale is $\tau_{\text {fast }} \sim t_{o} \sim c^{-1 / 2}$.

Analogously, if $t_{n} \gg t_{o}$ then $c^{1 / 2} \gg n$ and growing grains will overlap potential sites before these have nucleated, forcing the number of nucleating grains to decrease with time. As new grains still appear at every stage of the transformation, we expect approximately homogeneous nucleation, and correspondingly a KJMA exponent close to 3 . We term this situation slow nucleation. Comparing the radii of the grains with the mean distance among them we find the characteristic time of the process: 


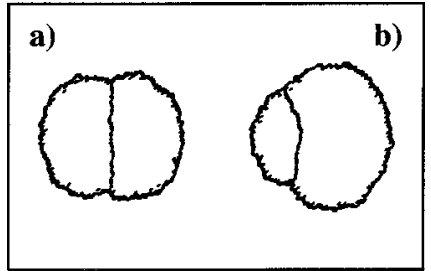

FIG. 3. Boundaries between two individual grains obtained from simulations with $g=0.5$ on a triangular lattice: (a) both grains nucleate at the same time, and (b) they nucleate at different times, yielding a curved interface.

$$
\begin{aligned}
\frac{1}{c^{1 / 2}\left[1-\exp \left(-n \tau_{\text {slow }}\right)\right]} & \simeq \frac{1}{\left(c n \tau_{\text {slow }}\right)^{1 / 2}} \\
& \sim r\left(\tau_{\text {slow }}\right)=\Omega \tau_{\text {slow }},
\end{aligned}
$$

and hence

$$
\tau_{\text {slow }} \sim \frac{1}{(c n)^{1 / 3}} .
$$

The important point, however, is the fact that between both limits we will find a wide range of KJMA exponents and grain size distributions, consistently with the experimental results.

\section{NUMERICAL RESULTS AND DISCUSSION}

\section{A. Isolated grain shapes}

An isolated grain, i.e., a grain completely surrounded by untransformed material, grows isotropically. Thus, in a continuum medium, the grain boundary is nearly a circumference. Nevertheless, the shape of such propagating interfaces in our model depends strongly on the underlying lattice. For example, in the limit case $g=1$, a grain growing in a square lattice is square shaped, whereas if growing in a triangular lattice it is hexagonal shaped. As the growth probability $g$ diminishes, the underlying lattice effects seem to vanish, and grains are approximately circular, with a rough boundary. In Figs. 1 and 2 we show the dependence of the grain shape on the growth probability, varying $g$ from 0.1 to 1 , on square and triangular lattices respectively. We see that for $g \leqq 0.4$ the shape of an isolated growing grain becomes practically independent of the lattice, whereas for larger values of $g$, the grain shape exhibits the influence of the lattice geometry. It is important to note that this does not occur when many

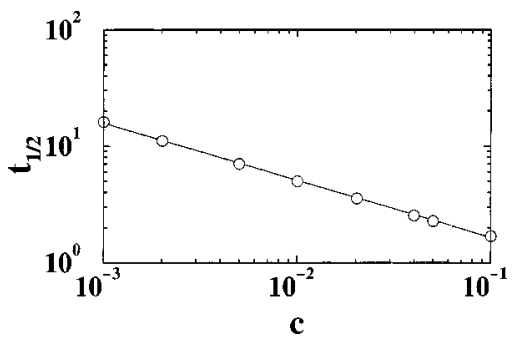

FIG. 4. $\log -\log$ plot of the characteristic time $t_{1 / 2}$ vs $c$ in the fast nucleation limit over a square lattice: $(\bigcirc)$ Simulation; solid line: power-law fit with slope $-0.50 \pm 0.01$.

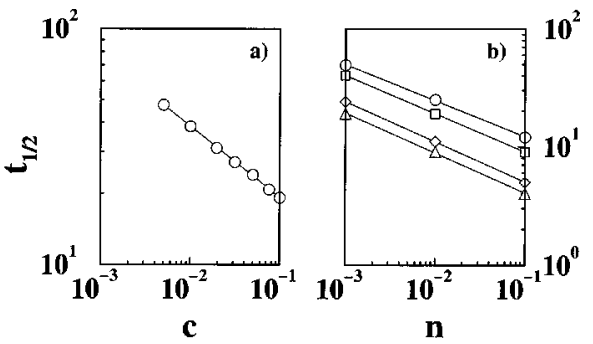

FIG. 5. $\log -\log$ plot of the characteristic time $t_{1 / 2}$ : (a) $(\bigcirc)$ Simulation value, solid line is a power-law fits with slope -0.32 \pm 0.01 ; (b) Symbols stand for simulation, solid lines are power fittings: $(\bigcirc) c=0.005$, slope: $0.34 \pm 0.02 ;(\square) c=0.01$, slope: $0.34 \pm 0.01 ;(\diamond) c=0.05$, slope: $0.32 \pm 0.01$; and $(\triangle) c=0.1$, slope: $0.31 \pm 0.02$.

grains grow simultaneously, as in this case the grain geometry is determined by the succesive impingement with its neighbors.

In connection with the last remark, it is interesting to consider another issue related to boundaries, namely that of boundaries between different grains. Let $r_{1}$ and $r_{2}$ be the radii of two circular grains; their boundary is then defined by the equation ${ }^{19}$

$$
r_{1}+v_{g} t_{1}=r_{2}+v_{g} t_{2},
$$

where $v_{g}$ is the growth velocity and $t_{1,2}$ the elapsed time since each grain nucleated. When grains started to grow at the same time $\left(t_{1}=t_{2}\right)$, the boundary is a straight line. Otherwise, it is a hyperbola. In Fig. 3 we plot two examples of interfaces in which, in spite of the fact that interfaces are noisy, both characteristic curves are revealed.

\section{B. Kinetics}

We have simulated $1000 \times 1000$ triangular and square lattices and averaged the outcome of 50 different realizations for each choice of parameters (characteristic simulation times are about 15 to 45 minutes in a Pentium II personal computer). The crystalline fraction ranges from 0 to 1 , so we define the typical simulation (or experimental) time as the time $t_{1 / 2}$ at which $X\left(t_{1 / 2}\right)=1 / 2$. As a check on our ideas, we have begun by verifying the dependence of this parameter on the time scales defined above. In Figs. 4 and 5 , we plot $t_{1 / 2}$ for different parameters in the fast and slow nucleation limits. A very good agreement is observed with the expected behavior of $t_{1 / 2} \sim \tau_{\text {fast }}$ and $t_{1 / 2} \sim \tau_{\text {slow }}$ discussed in Sec. II C.

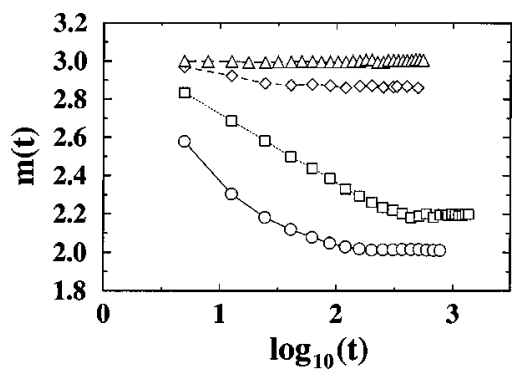

FIG. 6. Transient KJMA exponent vs $\log (t)$. Circles: $n=1$ and $c=0.001$; squares: $n=0.5$ and $c=0.005$; diamonds: $n=0.1$ and $c$ $=0.05$ and triangles: $n=0.01$ and $c=0.1 . g=1$ in all cases. 


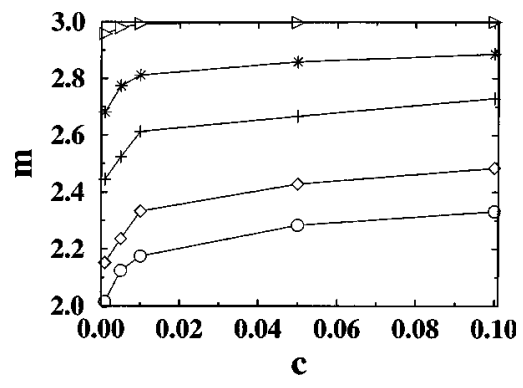

FIG. 7. KJMA exponent dependence on the concentration probability $c$ on a $1000 \times 1000$ triangular lattice. From top to bottom: $n=0.001, n=0.01, n=0.03, n=0.07$, and $n=1$.

Therefore, we can be confident that the expectations drawn above about the behavior of the model, based on theoretical considerations, will be fulfilled.

The first key feature to analyze relates to the crystallization kinetics as seen through KJMA plots. Our results show that those are not the straight lines predicted by the KJMA model: This can be best seen by looking at the transient KJMA exponent, defined as

$$
m(t)=\frac{d}{d(\log t)}\{\log [-\log (1-X)]\} .
$$

Figure 6 shows that the KJMA exponent always decreases from its initial value to an asymptotic, time-independent one; correspondingly and in agreement with the experiments, KJMA plots approach straight lines only at late times. We note that, in determining $m(t)$, care has to be taken from the computational point of view as in some cases the number of steps needed to complete the transformation is too short. In addition, it is necessary to remove the last few instants of the time evolution, as they exhibit large finite size effects. The asymptotic value is the one we take from simulations and the one plotted in Fig. 7 showing the dependence of the KJMA exponent with the potential site concentration $c$. Alternatively, Fig. 8 depicts the dependence of KJMA exponent on the nucleation probability $n$. We thus see that there is a large variability of the KJMA exponent, covering all the range between 2 and 3 in this two-dimensional case, that depends on the relationship between the nucleation probability $n$ (i.e., the nucleation rate) and the concentration of nucleation sites $c$. This result is a step beyond KJMA theory, and agrees with the fact that experiments offer very different results, with exponents between 2 and 3 .

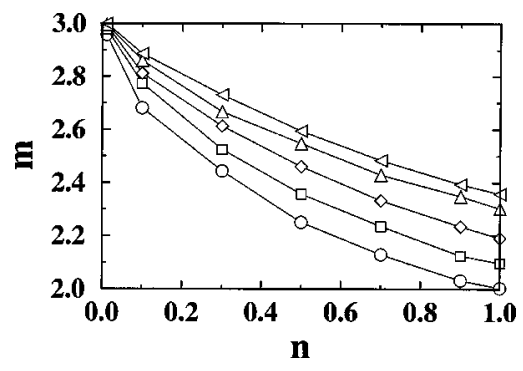

FIG. 8. KJMA exponent dependence on the nucleation probability $n$ on a $1000 \times 1000$ triangular lattice: $(\bigcirc) c=0.001 ;(\square) c$ $=0.005 ;(\diamond) c=0.01 ;(\triangle) c=0.05$; and $(\triangleleft) c=0.1$.

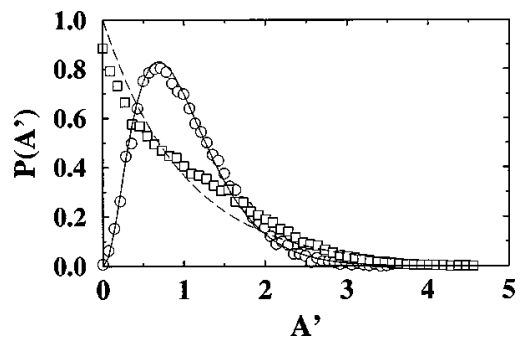

FIG. 9. Grain reduced area distribution: (O) Simulation with $n=1, g=1$, and $c=0.001 ;(\square)$ simulation with $n=0.001, g=1$, and $c=0.5$. Solid line: exact value from Eq. (15); dashed line: from Eq. (16).

\section{Grain area and grain diameter}

In order to further check the model results, we have to compare the grain size distributions with some well-accepted theoretical ones. Although these distributions are obtained phenomenologically, the agreement with experiments and simulations is very good. Under some assumptions about the mean number of neighbors of a nucleation center, Weaire et al. proposed a simple distribution for site saturation ${ }^{20}$

$$
P\left(A^{\prime}\right)=\left(A^{\prime}\right)^{\alpha-1} \alpha^{\alpha} \exp \left[-\alpha A^{\prime}\right] / \Gamma(\alpha),
$$

where $\alpha \simeq 3.65$, and $A^{\prime}=A / \bar{A}$ is the reduced area. In Fig. 9 we plot the normalized grain size distribution (circles) for different parameters for which $m \simeq 2$, i.e., site saturation, and compare it with Eq. (15) (solid line).

Similarly, in the case of homogeneous nucleation, a simple (but not so accurate) expression has been proposed ${ }^{21}$

$$
P\left(A^{\prime}\right)=\exp \left[-A^{\prime}\right]
$$

Our model shows some slight deviations from this equation, as seen in Fig. 9. Interestingly, these are the same as in other model simulations ${ }^{21}$ and, in addition, we have to keep in mind the applicability limitations of Eq. (16). ${ }^{21}$ Therefore, we believe that the behavior displayed by our model is also fully satisfactory in this limit.

Once we have checked the validity of the model in the well-known limits, we report on the influence of the nucleation probability, $n$, and the potential site concentration, $c$, on the grain size distribution. In Fig. 10 we plot several grain size distributions when we pick both parameters along a line going from the slow to the fast nucleation limit. In so doing, we cross from an extended distribution to a stretched one, as we would expect in view of Eqs. (15) and (16).

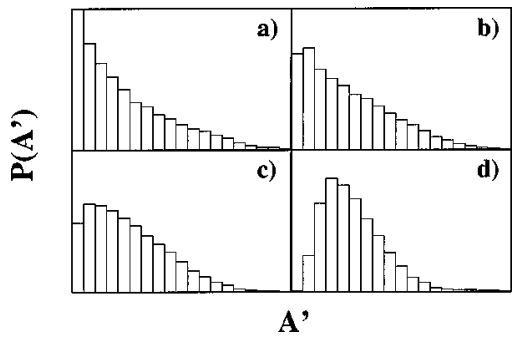

FIG. 10. Grain reduced area distribution. Simulation with: (a) $n=0.01$ and $c=0.1$; (b) $n=0.1$ and $c=0.05$; (c) $n=0.5$ and $c$ $=0.005$ and (d) $n=1$ and $c=0.001 . g=1$ in all cases. Horizontal axis ranges from 0 to 4 and vertical axes from 0 to 1 in four graphs. 


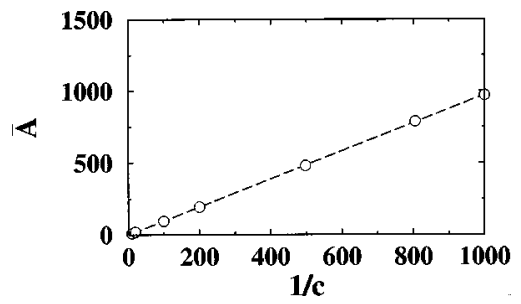

FIG. 11. Mean area vs inverse of the potential site concentration. (O) Simulation values. Dashed line is a linear fit.

Let us now turn to the issue of the mean grain size. As we have pointed out, in the fast nucleation limit the characteristic length scale is related to the mean potential site distance, $c^{-1 / 2}$ : In this case, we expect the mean grain diameter to be proportional to that scale. In Fig. 11 we show this linear dependence of the mean area on $c^{-1}$. On the contrary, in the slow nucleation limit, when the concentration $c$ is relatively large, the grains grow on an effective homogeneous medium. Roughly speaking, the mean distance among potential sites is so small that the grain radii is very soon larger than this distance. Thus, the characteristic length scale, $d$, is that of the grains when they impinge upon each other. As the grain radius grows linearly with time, we expect $d \sim t_{1 / 2}$, so $\bar{A}^{1 / 2}$ $\sim(c n)^{-1 / 3}$ and $\bar{A} \sim(c n)^{-2 / 3}$. Figure 12 confirms that this simple analysis is very accurate.

Finally, there are two questions we announced in Sec. II whose validation has been left postponed. We now address these points, beginning by that of the effect of the parameter $g$, which so far we have restricted to $g=1$. For every value of $g$, the growth rate, $f(g)$, defines a characteristic time related to the temporal scale at which the grains spread on the amorphous substrate. Thus, we expect that by rescaling the simulation time step $\tau \rightarrow f(g) \tau$ (with $f(g) \rightarrow \Omega$, as $g \rightarrow 1, \Omega$ being the geometrical coefficient introduced in Sec. II C), the mean grain size will depend only on the ratio $n / f(g)$. We have not been able to obtain an analytical expression for $f(g)$ but we can calculate it numerically for the required $g$, by growing an isolated grain. In Fig. 13 we show the excellent collapse of different effective diameter distributions for several couples $(n, g)$ with constant $n / f(g)$. This result shows that the outcome of the simulations reported here for $g=1$ truly represents, except for a factor, the model characteristics for other values of $g$.

The other pending question is related to the mean grain diameter. So far, we have discussed our results in terms of the mean grain area or the mean effective diameter size. To

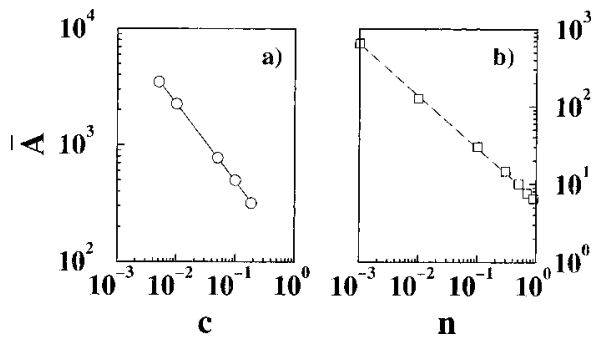

FIG. 12. $\log -\log$ plot of the mean area $\bar{A}$ : (a) (O) Simulation value; solid line: power-law fit with slope $-0.66 \pm 0.01$; (b) ( $\square$ ) simulation, dashed line: power-law fit with slope $-0.67 \pm 0.02$.

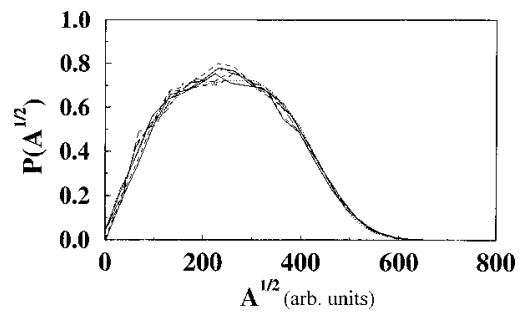

FIG. 13. Collapse of the grain effective diameter normalized distributions for eight $g$ values ranging from 0.05 to 1 .

verify whether the effective diameter distribution is the same as the real diameter distribution, which is computationally much more demanding, we have compared them in several cases. The comparison is shown in Fig. 14, by plotting the referred normalized distributions. The correlation between both sets of points is greater than $99.9 \%$, allowing us to conclude that the reports above in terms of areas carries over to the mean diameter picture without significant changes.

\section{Mean number of neighbors}

Some theoretical approaches to equilibrium crystallized configurations deal with the mean number of neighbors, $N_{n n},{ }^{21}$ or equivalently, considering the final product as a polygon tessellation of space, the mean number of sides of those polygons. If the material is divided in equal size hexagons, this distribution is $P\left(N_{n n}\right)=\delta\left(N_{n n}-6\right)$. In Fig. 15 we plot the numerical distribution of nearest neighbors for site saturation and homogeneous nucleation. The asymmetry and the variance of the mean number of neighbors are the main differences in both limits. The inset in Fig. 15 shows the mean number of neighbors and the corresponding changes in variance for different parameters. Clearly, the distribution spreads out and loses its symmetry in homogeneous nucleation. Furthermore, computing the mean number of neighbors against the nucleation time for all of the grains in the sample we find that the younger grains have less number of sides than the older ones, which explains this asymmetry. Hence, this distribution can be another element of comparison with experiments. We remark that secondary crystallization (or abnormal grain growth) is due to these deviations from the ideal configuration.

\section{E. Temperature and applicability of the model}

To conclude our analysis of heterogenous nucleation, we present some results of the influence of temperature in prod-

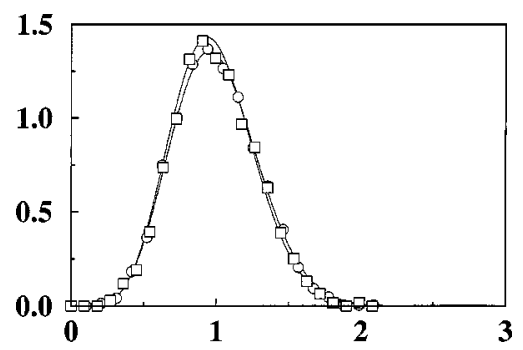

FIG. 14. Numerical comparison between normalized distributions of reduced grain diameter $(\bigcirc), d^{\prime}$, and reduced effective diameter, $\left(A^{\prime}\right)^{1 / 2}(\square)$. 


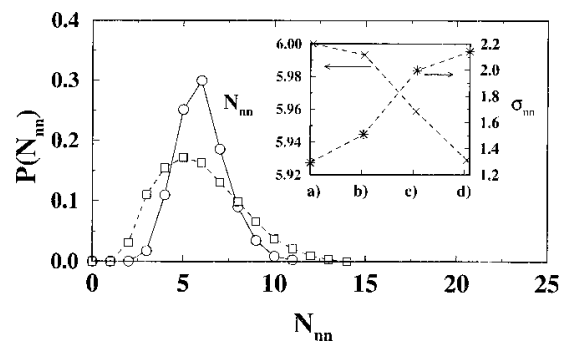

FIG. 15. Nearest-neighbor number normalized distribution. $(\bigcirc)$ $n=1, c=0.001$ (site saturation); $\square$ ) $n=0.01$ and $c=0.1$ (homogeneous nucleation). Inset: Mean number of neighbors, $N_{n n}$, and its variance, $\sigma_{n n}$ with: (a) $n=0.01$ and $c=0.1$; (b) $n=0.1$ and $c$ $=0.05$; (c) $n=0.5$ and $c=0.005$; and (d) $n=1$ and $c=0.001$.

uct properties. In addition, this will allow us to show that the model gives consistent results when realistic parameters are chosen to reproduce an actual material. The mean grain area in homogeneous nucleation of two-dimensional disks is given by the simple relation: ${ }^{19}$

$$
\bar{A} \sim\left(\frac{G_{0}}{N_{0}}\right)^{2 / 3},
$$

where $N_{0}$ and $G_{0}$ are the nucleation and growth rates, respectively. Identifying $N_{0}$ with $n$ and $G_{0}$ with $f(g)$, we have obtained similar results (see Sec. III C). As nucleation and growth are activated processes, we postulate an Arrheniuslike dependence of nucleation and growth probabilities:

$$
n \sim \exp \left[-E_{n} / k_{B} T\right], \quad f(g) \sim \exp \left[-E_{g} / k_{B} T\right] .
$$

In homogeneous nucleation, as we have reported, we can redefine $n$ and $g$ to set $g=1$; hence, the temperature is introduced in our model by means of the nucleation probability

$$
n \rightarrow n^{\prime}=n / f(g)=n_{0}^{\prime} \exp \left[-\left(E_{n}-E_{g}\right) / k_{B} T\right],
$$

and $g=1$.

As an example, if we want to model nondendritic Si crystallization, we may use the experimental activation energies: ${ }^{22} E_{n}=5.1 \mathrm{eV}$ and $E_{g}=3.2 \mathrm{eV}$. Then, $\bar{A}$ $\sim \exp \left[E_{a} / k_{B} T\right]$, where from Eq. (17) $E_{a}=2\left(E_{n}-E_{g}\right) / 3$ $\simeq 1.27 \mathrm{eV}$. In Fig. 16 we plot the mean grain size vs $1000 / T$. The slope gives $E_{a}=1.26 \pm 0.01 \mathrm{eV}$, which is consistent with the introduced values. Thus, the model provides a simple tool to analyze crystallization experiments: Setting the activation energies as the program input, we just have to choose a realistic value of $n_{0}^{\prime}$ (e.g., in terms of the final number of grains) and tune the degree of heterogeneities, $c$, in order to compare with the experiments.

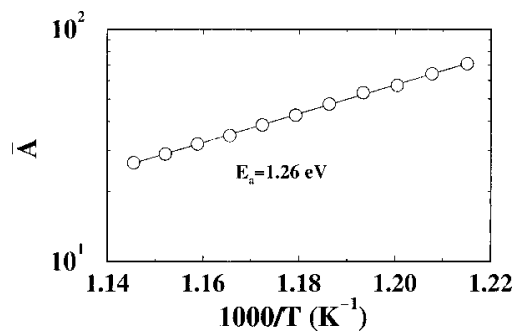

FIG. 16. Self-consistency of Eq. (19) with $c=1$ (homogeneous nucleation). ( $\bigcirc)$ Simulation; solid line: exponential fit which gives an activation energy $E_{a}=2\left(E_{n}-E_{g}\right) / 3=1.26 \pm 0.01$, consistent with $E_{n}=5.1 \mathrm{eV}$ and $E_{g}=3.2 \mathrm{eV}$.

\section{CONCLUSIONS}

As we have seen, the model proposed in this paper provides very accurate and detailed spatial and temporal information about the system evolution: Crystalline fraction, mean grain area, KJMA exponent, or mean number of neighbors. The main features observed in experiments, such as noninteger KJMA exponents or different types of grain size distributions are very well reproduced by the model. We must conclude, then, that the model captures all the physical ingredients involved in the crystallization process: In particular, it points out to the inhomogeneity of the nucleation phenomenon (which can arise because of the structure of the amorphous material itself, or because of defects at the substrate-material interface, for instance) as the key feature governing the crystallization kinetics and the resulting grain textures. In view of this, we propose this model, very unexpensive in terms of computing time, as a versatile way to incorporate other physical ingredients as boundary migration, preferential grain growth or diffusion-controlled growth, which will be the aim of further work. Finally, from the experimental perspective, it has to be mentioned that the model should be able to explain and predict some results. Predictions can be made by means of $n^{\prime}$, controlled by changing the annealing temperature (see Sec. III E), and $c$ by ion implantation of nucleation centers, or by some induced impurities or defects on the sample substrate. Some ordered distributions of defects can be induced by ion implantation with an appropriate mask, which can be trivially introduced in our model. These ideas call for further experimental work in order to confirm the validity of our model.

\section{ACKNOWLEDGMENTS}

This work was supported by CAM (Madrid, Spain) under Project No. 07N/0034/98 and by DGESIC (Spain) under Project No. PB96-0119. The authors wish to thank T. Rodríguez, A. Rodríguez, J. Olivares, and C. Ballesteros for simulating dicussions on experimental issues.
*Also at Universidad Pontificia de Comillas, E-28015 Madrid, Spain.

${ }^{1}$ J. S. Im and R. S. Spolisi, MRS Bull. 21 (3), 39 (1996).

${ }^{2}$ R. Bergmann, G. Oswald, M. Albrecht, and J. H. Werner, Solid State Phenom. 51-52, 515 (1996).

${ }^{3}$ K. Zellama, P. Germain, S. Squelard, J. C. Bourgoin, and P. A. Thomas, J. Appl. Phys. 50, 6995 (1979).

${ }^{4}$ H. V. Atkinson, Acta Metall. 36, 469 (1988).
${ }^{5}$ R. D. Doherty, Prog. Mater. Sci. 42, 39 (1997).

${ }^{6}$ R. Sinclair, J. Morgiel, A. S. Kirtikar, I. W. Wu, and A. Chiang, Ultramicroscopy 51, 41 (1993).

${ }^{7}$ C. W. Price, Acta Metall. Mater. 38, 727 (1990), and references therein.

${ }^{8}$ A. E. Kolmogorov, Akad. Nauk. SSSR Izv. Ser. Mat. 1, 355 (1937); W. A. Johnson and R. F. Mehl, Trans. Am. Inst. Min., Metall. Pet. Eng. 135, 416 (1939); M. Avrami, J. Chem. Phys. 7, 
103 (1939).

${ }^{9}$ V. Erukhimovitch and J. Baram, Phys. Rev. B 50, 5854 (1994); 51, 6221 (1995).

${ }^{10}$ E. Pinedau and D. Crespo, Phys. Rev. B 60, 3104 (1999).

${ }^{11}$ V. Sessa, M. Fanfoni, and M. Tomellini, Phys. Rev. B 54, 836 (1996).

${ }^{12}$ M. Fanfoni and M. Tomellini, Phys. Rev. B 54, 9828 (1996).

${ }^{13}$ C. W. Van Siclen, Phys. Rev. B 54, 11845 (1996).

${ }^{14}$ R. W. Cahn, J. Inst. Met. 76, 121 (1949).

${ }^{15}$ R. W. Cahn, Proc. Phys. Soc., London, Sect. A 63A, 323 (1950).

${ }^{16}$ P. A. Beck, TMS AIME 194, 979 (1972).
${ }^{17}$ M. Castro, F. Domínguez-Adame, A. Sánchez, and T. Rodríguez, Appl. Phys. Lett. 75, 2205 (1999).

18 In polycrystalline silicon, it has been observed that direction (111) is preferred throughout the transformation, and that it mainly nucleates at the interface with the underlying substrate.

${ }^{19}$ H. J. Frost and C. V. Thompson, Acta Metall. 35, 529 (1987).

${ }^{20}$ D. Weaire, J. P. Kermode, and J. Wejchert, Philos. Mag. B 53, L101 (1986).

${ }^{21}$ P. A. Mulheran, Acta Metall. Mater. 42, 3589 (1994).

${ }^{22}$ Y. Masaki, P. G. LeComber, and A. G. Fitzgerald, J. Appl. Phys. 74, 129 (1993). 